\title{
Assistência de enfermagem diante dos agravantes causados pela endometriose
}

\author{
Nursing care in view of the aggravations caused by endometriosis \\ Asistencia de enfermería ante los agravantes causados por la endometriosis
}

Recebido: 28/10/2021 | Revisado: 06/11/2021 | Aceito: 11/11/2021 | Publicado: 17/11/2021

\author{
Laís de Barros Xavier \\ ORCID: https://orcid.org/0000-0002-5924-1162 \\ Universidade Paulista, Brasil \\ E-mail: laxavier43@gmail.com \\ Maria Luiza Rêgo Bezerra \\ ORCID: https://orcid.org/0000-0002-3336-7760 \\ Universidade Paulista, Brasil \\ E-mail: marialuizaregobezerra@gmail.com
}

\begin{abstract}
Resumo
Objetivo: As mulheres acometidas pela endometriose que levam consigo o fator de infertilidade possui uma queda no seu estilo de vida pessoal e social, ocasionado pela tristeza e angustia. Dessa forma, o objetivo desse estudo será analisar os impactos psicológicos, e a assistência de enfermagem nas mulheres acometidas pela endometriose. Método: Compõese de uma revisão integrativa de literatura, que permite considerar a avaliação da bibliografia presente, com a aplicabilidade de extensos temas, amostras, estudos que emergem seus resultados de maneira significativa na prática. Resultado: Dentre os estudos selecionados para a pesquisa, oito dos artigos que foram citados no texto foram publicados em 6 periódicos diferentes, nos anos de 2010 a 2021. Conclusão: Conclui-se com esse estudo, que a endometriose é uma doença que se aloja e causa inúmeros transtornos na qualidade de vida das mulheres. Assim, espera-se contribuir para que a assistência de enfermagem no manejo da endometriose, possa estar buscando novos conhecimentos quanto ao tratamento desta doença, tendo em vista a importância da prática de novas pesquisas referente a temática abordada. Palavras-chave: Endometriose; Cuidados de enfermagem; Infertilidade feminina; Saúde da mulher; Ansiedade.
\end{abstract}

\begin{abstract}
Objective: Women affected by endometriosis who carry the infertility factor have a decline in their personal and social lifestyle, caused by sadness and anguish. Thus, the aim of this study will be to analyze the psychological impacts and nursing care in women affected by endometriosis. Method: It consists of an integrative literature review, which allows considering the evaluation of the present bibliography, with the applicability of extensive themes, samples, studies that significantly emerge their results in practice. Results: Among the studies selected for the research, eight of the articles that were cited in the text were published in 6 different journals, from 2010 to 2021. Conclusion: It is concluded with this study that endometriosis is a disease that lodges itself and it causes numerous disorders in women's quality of life. Thus, it is expected to contribute so that nursing care in the management of endometriosis may be seeking new knowledge regarding the treatment of this disease, considering the importance of practicing new research on the topic addressed.
\end{abstract}

Keywords: Endometriosis; Nursing care; Female infertility; Women's health; Anxiety.

\section{Resumen}

Objetivo: Las mujeres afectadas por endometriosis portadoras del factor infertilidad presentan un deterioro en su estilo de vida personal y social, provocado por la tristeza y la angustia. Así, el objetivo de este estudio será analizar los impactos psicológicos y cuidados de enfermería en mujeres afectadas por endometriosis. Método: Consiste en una revisión integradora de la literatura, que permite considerar la evaluación de la bibliografía presente, con la aplicabilidad de temas extensos, muestras, estudios que emergen significativamente de sus resultados en la práctica. Resultados: Entre los estudios seleccionados para la investigación, ocho de los artículos que fueron citados en el texto fueron publicados en 6 revistas diferentes, de 2010 a 2021. Conclusión: Se concluye con este estudio que la endometriosis es una enfermedad que se aloja y causa numerosos trastornos en la calidad de vida de la mujer. Así, se espera contribuir para que los cuidados de enfermería en el manejo de la endometriosis busquen nuevos conocimientos sobre el tratamiento de esta enfermedad, considerando la importancia de practicar nuevas investigaciones sobre el tema abordado.

Palabras clave: Endometriosis; Cuidado de enfermera; Infertilidad femenina; La salud de la mujer; Ansiedad. 


\section{Introdução}

A endometriose consiste em um tecido fora da cavidade uterina, gerando uma resposta inflamatória. É uma patologia que cresce gradativamente, e atinge mulheres na fase reprodutiva, e seus sintomas podem alterar-se desde sangramentos menstruais anormal ou serem completamente assintomáticos. Descrita por Sampson em 1927, a teoria da menstruação retrograda, é a mais considerada na atualidade. (Tsamantioti \& Mahdy, 2021)

Apesar de ser comum, acomete mais mulheres em idade reprodutiva, manifestando de maneira assintomática, ou acompanhada de determinados sintomas como dor pélvica crônica, dismenorreia, dores intestinais e urinarias além de uma causa relevante, a infertilidade. (Cardoso et al., 2020) Essa patologia pode ser diagnosticada em torno de 10 anos com o início dos sintomas, e a dor ocasiona consultas inespecíficas, ocasionando a demora no diagnóstico. (Gruber \& Mechsner, 2021)

Seu diagnóstico é realizado através de exame como transvaginal com preparo intestinal, e ressonância magnética da pelve. (Ribeiro et al., 2021) Juntamente com o exame histológico com confirmação laparoscópica é bastante eficaz, sendo possível a detecção de forma concisa, porém, um ultrassom de alta precisão possibilita a descoberta da doença precocemente. (Guardo, Shah, Cerana, 2019)

$\mathrm{Na}$ endometriose suas lesões variam de localizações, podendo aparecer nos ovários, trato intestinal, sistema urinário, pleura, pericárdio e sistema nervoso central. (Araújo \& Passos, 2020). Sua prevalência é de 10 - 15\% nas mulheres, dentre elas, $3 \%$ são mulheres em idade férteis, e cerca de 50\% das mulheres acometidas, tem a causa de infertilidade. (Guardo et al., 2019) $\mathrm{Na}$ literatura mostra que após a menopausa pode regredir a doença, desta forma diminuindo a produção de estrógeno na mulher. (Araújo \& Passos, 2020)

Como tratamento, existe as possibilidades não invasiva como a terapia farmacológica em uso de analgésicos, terapia hormonal tendo como objetivo interromper o fluxo menstrual por meio de anticoncepcionais orais e também intervenções invasivas, como as cirurgias precisas ou radical com oscilação nos seus resultados. (Guardo et al., 2019)

A enfermagem tem papel fundamental no processo do cuidado dessas mulheres, iniciado desde a triagem de maneira humanizada (acolhendo e mantendo sempre a disposição de escuta-las), bem como o acesso a informações e orientações para alivio das dores e servindo como um apoio psicológico, tendo em vista o nosso manejo em acolher, escutar e prestar o cuidado. (Oliveira et al., 2018) Mas, muito além disso, o atendimento das especialidades psicossociais é de grande importância, pois, temse a existência de um leque mais abrangente do que apenas as necessidades biológicas, assim, facilitam o entendimento da doença ao longo dos tempos e melhoria da qualidade de vida. (Souza et al., 2019)

Dada a natureza crônica da endometriose, as mulheres passam por dificuldade de identificar a causa relacionada aos seus sintomas, e por consequência não obter um tratamento especifico, causando um impacto potencial na fertilidade e nas relações intimas com seus parceiros, propiciando um isolamento que atinge negativamente seus aspectos de vida, resumindo ainda em que a vida dessas mulheres se torna tão dolorosas, não somente com a dor psicológica, mais em suas relações intimas, onde o prazer é substituído pela dor e desconforto durante as relações sexuais, (Araújo \& Passos, 2020). com os desconfortos e as dores, a procura pelo parceiro diminui, cerca de 32,26\%. (Baetas et al., 2021)

Neste cenário, as mulheres acometidas pela endometriose que levam consigo o fator de infertilidade possui uma queda no seu estilo de vida pessoal e social, ocasionado pela tristeza e angustia, e por consequência seus parceiros também são afetados, embora exista outras possibilidades como a fertilização in vitro. Dessa forma, o objetivo desse estudo será analisar os impactos psicológicos, e a assistência de enfermagem nas mulheres acometidas pela endometriose. 


\section{Metodologia}

Compõe-se de uma revisão integrativa de literatura, que permite considerar a avaliação da bibliografia presente, com a aplicabilidade de extensos temas, amostras, estudos que emergem seus resultados de maneira significativa na prática. Juntamente com identificações de informações imprescindíveis, para a busca dos estudos na literatura, permitindo estudos experimentais e não-experimentais, para que se obtenha compreensão eficaz do estudo abordado. Gerando conceitos complexos, de teorias e problemas de saúde para a enfermagem. (Souza, Silva \& Carvalho, 2010)

Foram utilizando os bibliográficos eletrônicos do banco de dados Publisher Medline (Pubmed), Portal Regional da BVS, Bases de Dados de Enfermagem (BDENF) e Scientific Electronic Library Online (SciELO), para analisar sobre a literatura existente, avaliando sobre o tema de Endometriose e a assistência de Enfermagem, baseado em evidências cientificas para contribuir na prática da Enfermagem.

\subsection{Coleta e organização dos dados}

Foram utilizados para a formação deste estudo, pesquisa nas bases de dados com revisões de 2011 a 2021, filtrados em assunto principal: Endometriose e Infertilidade feminina; Em idiomas: Português e Inglês.

Para a coleta dos dados foram seguidos os passos, a saber: formulação da pergunta norteadora, pesquisa nas bases de dados sobre o tema a ser abordado; formulação da pergunta norteadora; segregação e analise dos artigos; organização e formação do trabalho.

Na coleta de dados foram encontrados 2.440 artigos com a aplicação dos métodos de inclusão e exclusão, sendo artigos citáveis, de 2011 a 2021, nos idiomas Português e Inglês. Foram usados os descritores cruzados com os marcadores boleanos “and” Endometriose; cuidados de enfermagem; Infertilidade Feminina; Saúde da Mulher; Ansiedade, retirado dos Descritores em Ciências da saúde (DeCS), Pubmed e Scielo. Destes, aplicando as estratégias de inclusão, para artigos dos últimos 10 anos, nos idiomas citados, mostrou um total de 771 artigos. E foram excluídos os artigos que não se relacionava com o tema, e de maneira não gratuita, totalizando 41 artigos.

A coleta foi realizada em 2021, utilizando os descritores e palavras-chave: Endometriose, Cuidados de Enfermagem, Infertilidade Feminina, Ansiedade, endometriosis, infertility, anxiet, psychological factors, nursing care. Utilizaram-se os descritores e palavras-chave procedendo para as buscas descritas (Quadro 1):

Quadro 1: Estratégia de Busca. Brasília - DF, 2021.

\begin{tabular}{cl} 
SciELO & $\begin{array}{l}\text { (endometriosis or infertility or infertility and anxiety) and (infertility and } \\
\text { psychological factors or infertility and nursing care) }\end{array}$ \\
PUBMED & $\begin{array}{l}\text { (endometriosis or retrograde menstruation or) and (endometriosis and } \\
\text { nursing) }\end{array}$ \\
\hline BVS & $\begin{array}{l}\text { (endometriosis or infertility or infertility and anxiety) and (infertility and } \\
\text { psychological factors or infertility and nursing care) }\end{array}$ \\
\hline
\end{tabular}

Fonte: Autoras (2021).

Método de inclusão foram artigos originais publicado de maneira livre e gratuita nos idiomas Português e Inglês dos últimos dez anos nas bases de dados selecionadas. E a exclusão se deu para artigos que não tinha relação com a temática pesquisada e que obtiveram pesquisas repetidas nas bases de dados, artigos que não são disponibilizados de forma gratuita, pesquisa esta, se enquadrando dentro do conselho de ética, como exibe na (Figura 1): 
Figura 1: Fluxograma, Coleta e Análise dos Dados. Brasília - DF, 2021.

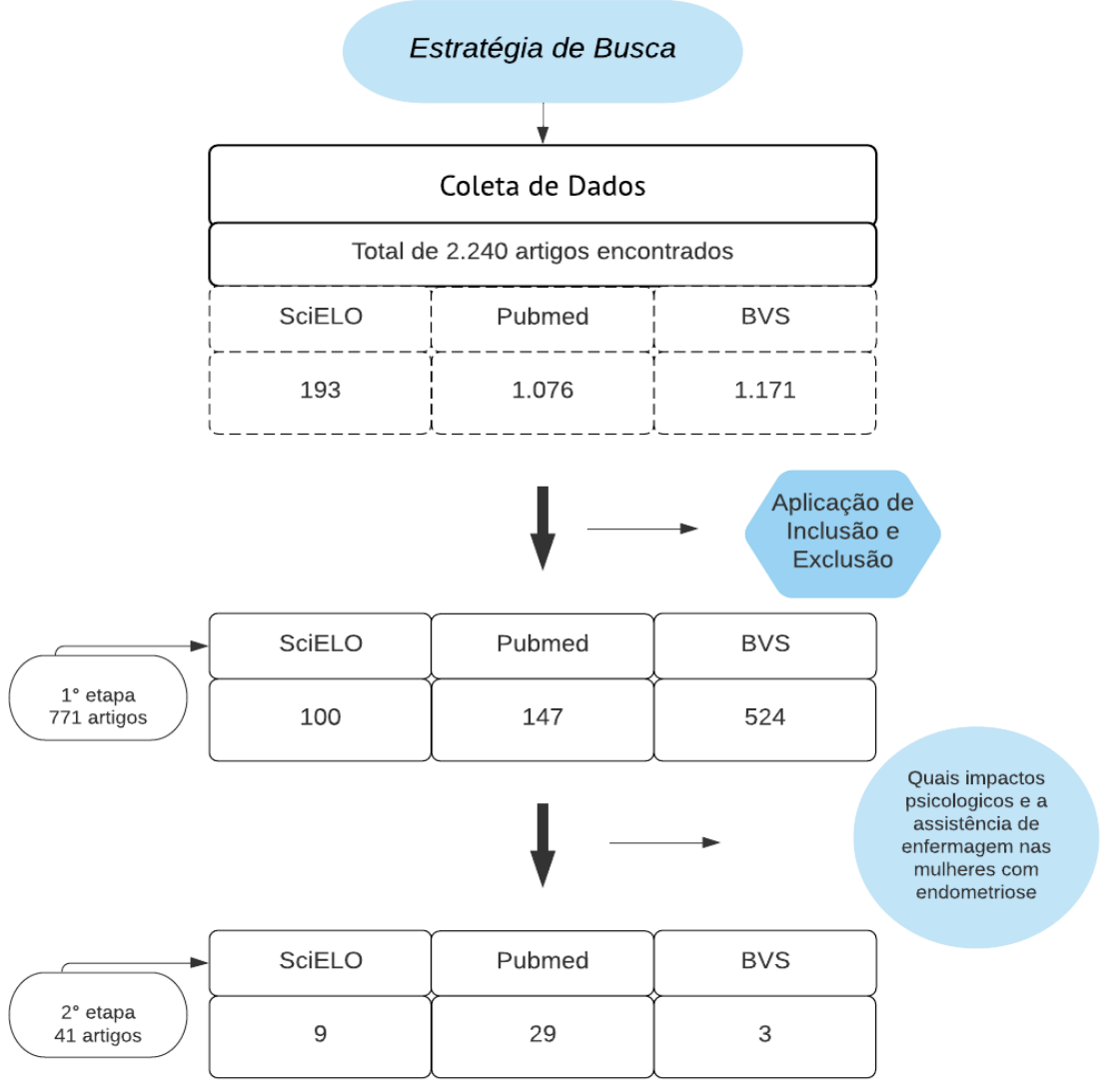

Fonte: Autores (2021).

\section{Resultados}

Apresenta-se a seguir o Quadro 2, onde irá mostrar os estudos selecionados que estão exposto no artigo, considerando informações de Título, Periódicos, Ano, Método e os Níveis de evidencias. Os artigos analisados foram organizados de forma a favorecer a melhor leitura dos resultados.

Quadro 2: Características do estudo abordado.

\begin{tabular}{|c|c|c|c|c|}
\hline Titulo & Periódico & Ano & Método & Nível de evidência \\
\hline Endometriose $^{(1)}$ & $\begin{array}{l}\text { Publisher Medline } \\
\text { (Pubmed) }\end{array}$ & 2021 & $\begin{array}{c}\text { Estudo de revisão de } \\
\text { literatura }\end{array}$ & IV \\
\hline $\begin{array}{l}\text { Perfil epidemiológico de mulheres } \\
\text { com endometriose: um estudo } \\
\text { descritivo retrospectivo }\end{array}$ & SciELO & 2020 & $\begin{array}{l}\text { Estudo Descritivo } \\
\text { retrospectivo }\end{array}$ & $\mathrm{V}$ \\
\hline $\begin{array}{l}\text { Tratamento de mulheres afetadas pela } \\
\text { endometriose: estamos avançando } ?^{(3)}\end{array}$ & $\begin{array}{c}\text { Jornal de Endometriose } \\
\text { e Distúrbios da dor } \\
\text { Pélvica }\end{array}$ & 2019 & Estudo de revisão & $\mathrm{V}$ \\
\hline $\begin{array}{l}\text { Endometriose: Contribuição da } \\
\text { Enfermagem Em Seu Cuidado }^{(4)}\end{array}$ & $\begin{array}{l}\text { Revista JRG de Estudos } \\
\text { Acadêmicos }\end{array}$ & 2020 & $\begin{array}{c}\text { Revisão sistematizada de } \\
\text { literatura } \\
\end{array}$ & II \\
\hline $\begin{array}{l}\text { A importância do acolhimento da } \\
\text { equipe de enfermagem no tratamento } \\
\text { da endometriose }{ }^{(5)}\end{array}$ & GEP NEWS & 2018 & $\begin{array}{c}\text { Estudo exploratório- } \\
\text { descritivo - revisão de } \\
\text { literatura simples } \\
\end{array}$ & IV \\
\hline $\begin{array}{c}\text { Endometriose e a qualidade de vida } \\
\text { das mulheres acometidas }{ }^{(6)}\end{array}$ & $\begin{array}{l}\text { Revista Eletrônica } \\
\text { Acervo Cientifico }\end{array}$ & 2021 & $\begin{array}{c}\text { Estudo transversal e } \\
\text { qualitativo }\end{array}$ & III \\
\hline $\begin{array}{l}\text { Revisão integrativa: o que é? Como } \\
\text { fazer isso? }\end{array}$ & SciELO & 2010 & Revisão integrativa & IV \\
\hline
\end{tabular}

Fonte: Autores (2021). 


\section{Descrição dos 8 estudos resultante da pesquisa:}

Dentre os estudos selecionados para a pesquisa, oito dos artigos que foram citados no texto foram publicados em 6 periódicos diferentes, nos anos de 2010 a 2021. Onde 1 é na PubMed, 2 são na SciELO, 1 na Revista JRG de Estudos Acadêmicos, 1 na GEP News e 1 na Revista Eletrônica Acervo Cientifico.

\section{Discussão}

\section{Os impactos psicológicos nas mulheres acometidas pela endometriose}

Segundo Sampson (1927), a teoria da menstruação retrograda constitui o eixo mais notado, que incide aparentemente em que, ao invés de seu fluxo menstrual descamar a parede do endométrio e ser eliminado, ele faz o ciclo contrário pelas trompas de falópio, atingindo outras extremidades. Dessa maneira, pequenos rastros do endométrio (sangue) se alojam, assim, gerando uma inflamação no local acometido. (Sampson, 1927)

A endometriose vem ocasionando grandes impactos na saúde da população feminina, pois ela interfere no ciclo da vida da maior parte da desta população. Carregando em si uma etiologia desconhecida, os sintomas podem percorrer ao longo da vida das mulheres de maneira imperceptível, ou de formas que pareça natural sem ser considerada que há algo de errado, o que acaba mascarando a patologia. (Baetas et al., 2021)

Esta patologia pode ser evidenciada através de sinais e sintomas bastante comuns, como dismenorreia, dor pélvica, dor abdominal, causando grandes desconfortos na rotina da mulher. Embora sintomas comuns, a endometriose causa um grande impacto na qualidade de vida das mulheres, a infertilidade. (Silva et al., 2020)

A infertilidade é o contribuinte mais difícil de ser aceito, pois, o sentimento é como se não pudesse dar continuidade ao ciclo da vida. Um filho é considerado como de alto valor, e com a incapacidade de concebe-lo o casal se sente incapaz, imperfeitos, assim gerando conflitos entre si, e problemas de maior agravamento como depressão, ansiedade, estresses. (Farinati, Rigoni \& Müller, 2006)

Detectar a doença precocemente ajuda no atraso da doença, e evita possíveis conflitos que surgiria ao longo do progresso da doença. Nem sempre isso é possível pelos sintomas apresentados serem frequentemente comuns e assim, confundidos com outra causa. Determinadas mulheres quando descobrem a patologia relatam estar aliviadas, outras descrevem que sentem raiva pela demora dos resultados. (Culley et al., 2013)

Seu tratamento não tem cura, e sim alívio dos sintomas. Os anticoncepcionais orais (ACO), são utilizados como primeira opção para tratamento, por serem de fácil acesso, e seu uso pode ser prolongado. Pois reduzem as taxas de estrogênio no organismo, retardando a fórmula da doença. No entanto essa redução faz com que apareça sintomas como instabilidade do humor. (Nácul \& Spritzer, 2010) E o tratamento deve ser feito de maneira individual e interdisciplinar, uma vez que sinais e sintomas são individuais de cada mulher. (Chapron et al., 2019)

Como tratamentos posteriores aos ACO, possuem os tratamentos que irão depender do nível da doença, tempo, e outros fatores associados. Incluídos, os de reprodução assistida e cirurgia para cauterizar focos das lesões. (Nácul \& Spritzer, 2010) Mas, com o uso de medicamentos pode ocorre efeitos colaterais, e os sintomas podem retornar devido à cessação da utilização dos ACO. (Mińko et al., 2021)

Além disso, mulheres que passam por enfrentamento da endometriose com a sintomatologia de dismenorreia, dor pélvica, dor abdominal, mostram-se com níveis de depressão, comparadas a mulheres que não demonstram tais sintomas. (Silva, Cunha, Neves, Mascarenhas \& Caroci-Becker, 2021) E um estudo realizado com 171 mulheres em tratamento da endometriose no período entre abril - agosto de 2014, ressaltou que pacientes com a patologia que levam a vida de maneira leve e com persistência, apresentam poucos sintomas de estresse e depressão. (Donatti, Ramos, Andres, Passman \& Podgaec, 2017) 


\section{A assistência de enfermagem diante as mulheres com Endometriose}

A enfermagem liga no contexto da escuta e do cuidado, onde se colocam a disposição para que as mulheres se sintam acolhidas. Pois muitas já estão exaustas com respostas superficiais de que suas dores sejam "normais", e a enfermagem tem papel fundamental nessa trajetória de acolher e informar essas pacientes para que elas se sintam abrigadas e que possam diminuir o cansaço físico e psicológico. (Brilhante, 2019)

Vale ressaltar, que é importante para que os profissionais da enfermagem tenham conhecimento dos sinais, sintomas e aspectos desta patologia, colocando em prática promoção de ações em saúde para informar a importância da rede de apoio familiar, social e até mesmo o interpessoal. Seguindo protocolos voltados ao acolhimento da doença, e não apenas fornecendo os cuidados gerais, dificultando assim a descoberta precoce da doença e transtornos psicológicos. (Araújo \& Passos, 2020)

E também, um papel não menos importante da equipe de enfermagem, é na identificação das dores que antecedem os sinais e sintomas da doença, realização da triagem e exames ginecológicos. Onde facilita o diagnóstico da doença, e continuamente traz o alívio, pois retira o peso de não saber a causa existente por trás daquela dor. Agir de forma hábil para o diagnóstico da endometriose, é de forma positiva, pois melhora os resultados na vida das mulheres. (Araújo \& Passos, 2020)

\section{Conclusão}

Conclui-se com esse estudo, que a endometriose é uma doença que se aloja e causa inúmeros transtornos na qualidade de vida das mulheres. Enquanto a causa da doença não for descoberta, as mulheres tendem a continuar com as dores frequentemente, ocasionando estresse emocional, psicológico, e com limitação na relação sexual, já que a dor durante a relação é extremamente desconfortável e doloroso, ainda neste contexto, dificultando relações com seus parceiros.

Existe os tratamentos clinico ou cirúrgico, o que irá definir o tratamento será da localização, extensão da doença e sintomatologia, e diagnostico deve ser feito pelo médico, os mais frequentes são com o uso de ACO e dependendo dos hábitos de vida dessas mulheres, a doença tem um maior potencial de se manifestar. Os focos da endometriose podem migrar para todo o organismo humano feminino, e com a cirurgia tenta-se retirar o máximo dos focos, mas esses focos podem retornar.

A enfermagem tem papel de suma importância, pois deve se manter um vínculo de confiança com a paciente para que a mesma possa desabafar e se sentir tranquilizada. Já que, muitas têm seus sentimentos descartados, pois, quando se diz ter fortes dores antecedentes a menstruação ou durante as relações sexuais com seus parceiros, isso é o considerado "normal”, "é só uma cólica".

O que ainda falta, é que os profissionais da área da saúde possam entender sobre a patologia, e conhecer os protocolos de atendimento a essas mulheres, pois a enfermagem tem o contato de imediato com essas pacientes. Deve-se adotar meios de comunicação para que as mulheres acometidas possam ser direcionadas a atenção para aquela história clínica, fazendo então que diminua o tempo de espera para a descoberta da causa e começar o tratamento clinico adequado.

Neste estudo, espera-se contribuir para que a assistência de enfermagem no manejo da endometriose, possa estar buscando novos conhecimentos quanto ao tratamento desta doença. Tendo em vista, a importância da pratica de novas pesquisas referente a temática abordada.

\section{Referências}

Araújo, G. V. \& Passos, M. A. N. (2020). Endometriose: Contribuição Da Enfermagem Em Seu Cuidado. Revista JRG De Estudos Acadêmicos.

Baetas, B. V., Bretas, V. B, Maziviero, C. M., Moraes, G. Z., Rodrigues, L. T. S., Zanluchi, A. \& Judice, W. A. S. (2021). Endometriose e a qualidade de vida das mulheres acometidas. Revista Eletrônica Acervo Científico. 19(1): 59-67

Brilhante, A. V. M. et al. (2019) Narrativas autobiográficas de mulheres com endometriose: que fenômenos permeiam os atrasos no diagnóstico? Physis: Revista de Saúde Coletiva. 29(3). 
Research, Society and Development, v. 10, n. 15, e41101522447, 2021

(CC BY 4.0) | ISSN 2525-3409 | DOI: http://dx.doi.org/10.33448/rsd-v10i15.22447

Cardoso, J. V., Machado de Silva, M. C., Berardo, P. T., Ferrari, R., Abrão, M. S. \& Perini, J. A. (2020) Epidemiological profile of women with endometriosis: a retrospective descriptive study. Revista Brasileira de Saúde Materno Infantil. 20(4),1057-67. FapUNIFESP (SciELO).

Chapron, C., Marcellin, L., Borghese, B. et al. (2019) Repensando os mecanismos, diagnóstico e tratamento da endometriose. Nat Rev Endocrinol 15, 666-682.

Culley, L., Law, C., Hudson, N., Mitchell, H., Denny, E., \& Raine-Fenning, N. (2017). Um estudo qualitativo do impacto da endometriose em parceiros masculinos. Reprodução humana (Oxford, Inglaterra), 32 (8), 1667-1673.

Donatti, L., Ramos, D. G., Andres, M. P., Passman, L. J., \& Podgaec, S. (2017). Patients with endometriosis using positive coping strategies have less depression, stress and pelvic pain. Einstein (Sao Paulo, Brazil), 15(1), 65-70.

Farinati, D. M., Rigoni, M. S. \& Müller, M. C. (2006). Infertilidade: um novo campo da psicologia da saúde. Estudos de Psicologia. $23(4)$, 433-9.

Gruber, T. M., \& Mechsner, S. (2021) Pathogenesis of Endometriosis: The Origin of Pain and Subfertility. 10 (6): 1381.

Guardo, F., Shah, M., Cerana, M. C., et al. (2019) Tratamento de mulheres afetadas pela endometriose: estamos avançando? Journal of Endometriosis and Pelvic Pain Disorders; 11 (2): 77-84.

Mińko, A., Turoń-Skrzypińska, A., Rył, A., Bargiel, P., Hilicka, Z., Michalczyk, K. \& Cymbaluk-Płoska, A. (2021). Endometriose - um problema multifacetado de uma mulher moderna. Jornal Internacional de Pesquisa Ambiental e Saúde Pública, 18 (15), 8177.

Nácul, A. P., \& Spritzer, P. M., (2010) Aspectos atuais do diagnóstico e tratamento da endometriose. Revista Brasileira de Ginecologia e Obstetrícia, 32(6), 298-307.

Oliveira, A. L. (2018). A Importância Do Acolhimento Da Equipe De Enfermagem No Tratamento Da Endometriose. GEP NEWS

Ribeiro, H. S. A. A., Paiva, A. M. F. de, Taliberti, B. da C. P., Gonçalves, A. L. L., Condes, R. P., \& Ribeiro, P. A. G. A. (2021) Problemas psicológicos vivenciados por pacientes com endometriose intestinal aguardando cirurgia. Revista Brasileira Ginecologia Obstetricia, 43 (09): 676-681.

Sampson, J. A., Peritoneal endometriosis due to the menstrual dissemination of endometrial tissue into the peritonealcavity. Am J Obstet Gynecol 1927; $14: 422-$ $469,1927$.

Silva, C. M., Cunha, C. F. da, Neves, K. R., Mascarenhas, V. H. A., \& Becker, A. C. (2021). Experiências das mulheres quanto às suas trajetórias até o diagnóstico de endometriose. Escola Anna Nery, 25(4), 12-26.

Silva, J. B., Gurian, M. B. F., Nonino, C. B., Poli-Neto, O. B., Nogueira, A. A., Reis, F. J. C. \& Rosa-e-Silva, J. C. (2020). Análise da composição corporal e intensidade de dor em mulheres com dor pélvica crônica secundária a endometriose. Rev. Bras. Ginecol. Obstret. 42(8): 55-69.

Souza, M. T., Silva, M. D. \& Carvalho, R. (2010) deIntegrative review: what is it? How to do it? Einstein, 8(1), 102-106.

Souza, T., Santos, N., Móta, J., da Silva, M., Silva, N. da, \& dos Santos, R. (2019). Papel da enfermagem frente a portadoras de endometriose e depressão. Revista de Enfermagem UFPE on line, 13(3), 811-818.

Tsamantioti, E. S., \& Mahdy, H. Endometriosis. Treasure Island (FL): StatPearls Publishing; 